\title{
Computing Upper-bounds of the Minimum Dwell Time of Linear Switched Systems via Homogeneous Polynomial Lyapunov Functions
}

\author{
G. Chesi ${ }^{1}$, P. Colaneri ${ }^{2}$, J. C. Geromel ${ }^{3}$, R. Middleton ${ }^{4}$, R. Shorten ${ }^{4}$
}

\begin{abstract}
This paper investigates the minimum dwell time for switched linear systems. It is shown that a sequence of upper bounds of the minimum dwell time can be computed by exploiting homogeneous polynomial Lyapunov functions and convex optimization problems based on linear matrix inequalities (LMIs). This sequence is obtained by adopting two possible representations of homogeneous polynomials, one based on Kronecker products, and the other on the square matrix representation (SMR). Some examples illustrate the use and the potentialities of the proposed approach. It is also conjectured that the proposed approach is asymptotically nonconservative, i.e. the exact minimum dwell time is obtained by using homogeneous polynomials with sufficiently large degree.
\end{abstract}

\section{INTRODUCTION}

This work deals with the stability of switched linear systems under a dwell time constraint. Problems in the design of switching control systems in which switching takes place "slowly" between system matrices, arise frequently in practice, see for instance [1]-[9]. In such problems, one is faced with determining the minimum time between switching (i.e. the dwell time) such that that exponential switching is maintained. It is well known that the computation of the exact minimum dwell time is demanding. In [10], an upper bound is computed on the basis of the norm of the transition matrices associated with the system matrices, and is further discussed in [11]. More recently, an alternative method based on convex optimization problems is presented in [12]. In this paper, the authors use linear matrix inequalities (LMIs) and one-parameter search techniques to compute a guaranteed dwell time. The inequalities generate a piecewise quadratic Lyapunov function $v(x)$, discontinuous at the switching instants $t_{k}$, but such that the sequence $v\left(x\left(t_{k}\right)\right)$, for $k=$ $0, \cdots, \infty$, converges uniformly to zero.

This paper extends the result in [12] by adopting homogeneous polynomial Lyapunov functions, which have been proposed in the context of time-varying systems [13]. A candidate Lyapunov function is looked for by using two possible representations, the first based on Kronecker products, and the second based on the square matrix representation (SMR) introduced in [14]. Both representations lead to sufficient conditions for stability of the switched linear system under

G. Chesi is with the Department of Electrical and Electronic Engineering, University of Hong Kong, contact: http://www.eee.hku.hk/ chesi

P. Colaneri is with the Department of Electronics and Information, Politecnico di Milano, contact: http://home.dei.polimi.it/colaneri

J. C. Geromel is with the School of Electrical and Computer Engineering, University of Campinas, contact: http://www.dt.fee.unicamp.br/ $/$ geromel

R. Middleton and R. Shorten are with the Hamilton Institute, National University of Ireland, contact: http://www.hamilton.ie/rick, http://www.hamilton.ie/bob a dwell time requirement via LMI feasibility tests, which are convex optimization problems with LMI constraints. A bisection search over a scalar parameter produces an upper bound of the minimum dwell time. The representation based on Kronecker products enjoys a more explicit formulation, while the one based on the SMR provides less conservative results. A number of examples illustrate the proposed approach, showing that the exact minimum dwell time can be often obtained.

The paper is organized as follows. In Section II the basic problem is formulated and some preliminary results are given. In Section III the proposed condition based on Kronecker products is derived. Section IV presents the formulation of this condition by adopting the SMR. Section $\mathrm{V}$ illustrates the proposed approach through a number of examples. Lastly, Section VI concludes the paper with some final remarks.

\section{Preliminaries}

The notation used throughout the paper is as follows: $\mathbb{R}$ : space of real numbers; $0_{n}$ : origin of $\mathbb{R}^{n} ; \mathbb{R}_{0}^{n}: \mathbb{R}^{n} \backslash\left\{0_{n}\right\} ; I_{n}$ : $n \times n$ identity matrix; $A^{\prime}$ : transpose of $A ; A>0$ : symmetric positive definite matrix $A ; A \otimes B$ : Kronecker product of matrices $A$ and $B$; s.t.: subject to.

We consider switched linear systems of the form

$$
\dot{x}(t)=A_{\sigma(t)} x(t)
$$

where $x(t) \in \mathbb{R}^{n}$, and $\sigma(t)$ is a switching signal taking values in a finite set $S=\{1,2, \ldots, M\}$. All matrices $A_{i}, i=$ $1,2, \ldots, M$, are assumed to be Hurwitz, and we characterize switching rules by saying that the signal $\sigma(t)$ orchestrates switching between the matrices $A_{1}, A_{2}, \ldots, A_{M}$.

In this work we impose a further restriction on the system class described by the above equation. More specifically, we impose restrictions on the set of admissible switching signals by defining the set

$$
D_{T}=\left\{\sigma(t): t_{k+1}-t_{k} \geq T\right\}
$$

where $t_{k}$ are the commutation instants and $T \geq 0$. The minimum dwell time problem is then to compute the minimum $T$ ensuring exponential stability of system (1) for all possible $\sigma(t) \in D_{T}$. We define this time as

$$
\begin{aligned}
T_{\min }= & \inf \{T \geq 0:(1) \text { is exponentially stable } \\
& \text { for all } \left.\sigma(t) \in D_{T}\right\} .
\end{aligned}
$$

Our starting point in this paper is the following Theorem that was given in [12] for guaranteeing a dwell time. 
Theorem 1 (see [12]): Assume that, for given $T>0$,

$$
\exists P_{i}:\left\{\begin{array}{l}
P_{i}>0 \quad \forall i \\
A_{i}^{\prime} P_{i}+P_{i} A_{i}<0 \quad \forall i \\
e^{A_{i}^{\prime} T} P_{j} e^{A_{i} T}<P_{i} \quad \forall i \neq j .
\end{array}\right.
$$

Then, the system is exponentially stable for every $\sigma(\cdot) \in D_{T}$.

The above result deserves a few remarks.

(i) For given Hurwitz matrices $A_{i}, i=1,2, \ldots, M$, there always exist $T>0$ such that (2) holds. Indeed, as $T$ goes to infinity, the third inequality reduces to $P_{i}>0$ and the feasibility of the second is guaranteed by Hurwitz stability of the matrices $A_{i}$.

(ii) If the inequalities are always satisfied for $T \rightarrow 0$, then, in the limit, it follows that $P_{j}-P_{i} \rightarrow 0$ so that the condition for quadratic stability is recovered, namely

$$
A_{i}^{\prime} P+P A_{i}<0, \quad \forall i
$$

where $P>0$ is the limit of $P_{i}$ as $T$ goes to 0 .

(iii) The function

$$
v(x, t)=x^{\prime} P_{\sigma(t)} x
$$

is a piecewise quadratic Lyapunov function for system (1) for every $\sigma(\cdot) \in D_{T}$.

(iv) Associated with a given a sequence $t_{k}, k=0,1, \ldots$, it is possible to write the discrete-time switched system

$$
\hat{x}(k+1)=F_{\hat{\sigma}(k)} \hat{x}(k)
$$

where

$$
\begin{gathered}
\hat{\sigma}(k)=\sigma\left(t_{k}\right), \quad \hat{x}(k)=x\left(t_{k}\right) \\
F_{\hat{\sigma}(k)}=e^{A_{\sigma\left(t_{k}\right)}\left(t_{k+1}-t_{k}\right)} .
\end{gathered}
$$

Then, this system is stable under arbitrary switching under the piecewise quadratic Lyapunov function

$$
w(\hat{x}, k)=\hat{x}^{\prime} P_{\hat{\sigma}(k)} \hat{x} .
$$

(v) Theorem 1 can be easily adapted to comply with possible state jumps in the system state. Indeed, assume that at each commutation instant $t_{k}$, the system state is reset according to the rule

$$
x\left(t_{k}\right)=S_{\sigma\left(t_{k}\right)} x\left(t_{k}^{-}\right) .
$$

Then stability in $D_{T}$ is guaranteed if there exist positive definite matrices $P_{i}$ satisfying

$$
\exists P_{i}:\left\{\begin{array}{l}
P_{i}>0 \quad \forall i \\
A_{i}^{\prime} P_{i}+P_{i} A_{i}<0 \quad \forall i \\
e^{A_{i}^{\prime} T} S_{j}^{\prime} P_{j} S_{j} e^{A_{i} T}<P_{i} \quad \forall i \neq j .
\end{array}\right.
$$

(vi) If the sufficient condition stated in Theorem 1 is feasible for $T$, then it holds also for $T+\xi$ for all $\xi \geq 0$.
The algorithm to find an upper bound of the minimum dwell time consists in finding the minimum value of $T$ such that (2) holds. Notice that this computation only involves the solution of a set of LMIs plus a line search over the parameter $T$. However, the sufficient condition stated in Theorem 1 is not necessary for stability in $D_{T}$. This means that a system can be stable in $D_{T}$ and no positive definite matrices $P_{i}$ exist satisfying (2). The reason is that the inequalities define a Lyapunov function $v(x)=x^{\prime} P_{\sigma(t)} x$, which is piecewise quadratic, whereas for stability in $D_{T}$, more complex Lyapunov functions are required. This latter observation is characterized by the following result which can be found in [15].

Theorem 2 (see [15]): The system is exponentially stable in $D_{T}$ if and only if there exist continuous functions $v_{i}(x)$ such that

$$
\left\{\begin{array}{l}
v_{i}(x)>0 \quad \forall x \neq 0_{n} \quad \forall i \\
\left.\frac{d v_{i}(x)}{d t}\right|_{\dot{x}=A_{i} x}<0 \quad \forall x \neq 0_{n} \forall i \\
v_{j}\left(e^{A_{i} T} x\right)<v_{i}(x) \quad \forall x \neq 0_{n} \forall i \neq j .
\end{array}\right.
$$

Let us observe that each $v_{i}(x)$ in Theorem 2 can be chosen homogeneous due to the fact that the system is linear.

\section{Conditions via Kronecker Products}

The idea exploited in this paper is to adopt homogeneous polynomial Lyapunov functions, which have the form

$$
v(x)=\sum_{\substack{i_{1}+\ldots+i_{n}=2 m \\ i_{1} \geq 0, \ldots, i_{n} \geq 0}} c_{i_{1}, \ldots, i_{n}} x_{1}^{i_{1}} \cdots x_{n}^{i_{n}}
$$

being $x=\left(x_{1}, \ldots, x_{n}\right)^{\prime} \in \mathbb{R}^{n}$ the function variable, $2 m$ the degree for a positive integer $m$, and $c_{i_{1}, \ldots, i_{n}} \in \mathbb{R}$ some coefficients.

One way to represent homogeneous polynomial Lyapunov functions is to use Kronecker products. Indeed, for any matrix (vector) $J$ and positive integer $i$ let us define the notation

$$
J^{\otimes i}= \begin{cases}J \otimes J^{\otimes i-1} & \text { if } i \geq 1 \\ 1 & \text { if } i=0 .\end{cases}
$$

Then, $v(x)$ in (6) can be rewritten as

$$
v(x)=\left(x^{\otimes m}\right)^{\prime} \Pi x^{\otimes m}
$$

for a suitable symmetric matrix $\Pi=\Pi^{\prime} \in \mathbb{R}^{n^{m} \times n^{m}}$ which contains the coefficients $c_{i_{1}, \ldots, i_{n}}$. Let $\mathcal{A}_{i, \otimes m} \in \mathbb{R}^{n^{m} \times n^{m}}$ be the matrix satisfying

$$
\frac{d x^{\otimes m}}{d t}=\frac{d x^{\otimes m}}{d x} A_{i} x=\mathcal{A}_{i, \otimes m} x^{\otimes m} \quad \forall x .
$$

It turns out that

$$
\mathcal{A}_{i, \otimes m+1}=\mathcal{A}_{i, \otimes m} \oplus A_{i}
$$


where the Kronecker sum of two matrices, say $X$ and $Y$ is defined as

$$
X \oplus Y=X \otimes I+I \otimes Y .
$$

Then, we have the following result.

Theorem 3: Assume that, for given $T>0$ and positive integer $m$,

$$
\exists \Pi_{i}:\left\{\begin{array}{l}
\Pi_{i}>0 \quad \forall i \\
\mathcal{A}_{i, \otimes m}^{\prime} \Pi_{i}+\Pi_{i} \mathcal{A}_{i, \otimes m}<0 \quad \forall i \\
e^{\mathcal{A}_{i, \otimes m}^{\prime} T} \Pi_{j} e^{\mathcal{A}_{i, \otimes m} T}<\Pi_{i} \quad \forall i \neq j .
\end{array}\right.
$$

Then, (1) is exponentially stable for every $\sigma(\cdot) \in D_{T}$. Proof. Suppose that (8) holds, and define

$$
v_{i}(x)=\left(x^{\otimes m}\right)^{\prime} \Pi_{i} x^{\otimes m} .
$$

Since

$$
\left(e^{A_{i} t} x\right)^{\otimes m}=e^{\mathcal{A}_{i, \otimes m} t} x^{\otimes m} \quad \forall t \geq 0
$$

it follows that (5) is satisfied thus implying that the system is exponentially stable for every $\sigma(\cdot) \in D_{T}$.

Remark 1: Let us observe that, for $m=1$, (8) coincides with (2).

Let us indicate with $T_{\otimes m}$ the smallest upper bound of $T_{\min }$ guaranteed by Theorem 3, i.e.

$$
T_{\otimes m}=\inf \{T \geq 0: \text { (8) holds }\} .
$$

The following result provides a key property of the condition (8), which allows one to calculate $T_{\otimes m}$ via a bisection search where at each iteration the condition (8) is tested.

Theorem 4: Assume that (8) holds for some $T>0$ and positive integer $m$. Then, (8) holds also for $T+\xi$ and $m$ for all $\xi \geq 0$.

Proof. Suppose that (8) holds, and define $v_{i}(x)=$ $\left(x^{\otimes m}\right)^{\prime} \Pi_{i} x^{\otimes m}$. Consider any $\xi \geq 0$. From the second inequality one has that

$$
v_{i}(x(\xi)) \leq v_{i}(x(0)) \quad \forall x(0)
$$

which implies that

$$
e^{\mathcal{A}_{i, \otimes m}^{\prime} \xi} \Pi_{i} e^{\mathcal{A}_{i, \otimes m} \xi} \leq \Pi_{i} .
$$

Pre- and post-multiplying the third inequality of (8) by $e^{\mathcal{A}_{i, \otimes m} \xi}$ and $e^{\mathcal{A}_{i, \otimes m}^{\prime} \xi}$ respectively, one gets that

$$
e^{\mathcal{A}_{i, \otimes m}^{\prime}(T+\xi)} \Pi_{j} e^{\mathcal{A}_{i, \otimes m}(T+\xi)}<\Pi_{i} .
$$

Therefore, the theorem holds.

The inequalities (8) are characterized by an important property for a fixed $T$. Indeed, denote by $\Pi_{i, \otimes r}$ the positive definite matrices satisfying (8) for a certain $T$ and $m=r$. Then, one can set

$$
\Pi_{i, \otimes 2^{k+1} m}=\Pi_{i, \otimes 2^{k} m} \otimes \Pi_{i, \otimes 2^{k} m}, \quad \forall k .
$$

This means that if (8) are feasible for $m=2^{k} r$, they are also feasible for $m=2^{k+1} r$. In conclusion, the sequence $T_{\otimes 2^{k} r}$, $k=0,1, \cdots$ is monotonically non-increasing with respect to $k$ and the limit

$$
T_{\otimes * r}=\lim _{k \rightarrow \infty} T_{\otimes 2^{k} r}
$$

exists. Of course, $T_{\otimes * r}$ is an upper bound of the minimum dwell time $T_{\min }$, for each $r$. As such

$$
T_{\otimes *}=\min _{r>0} T_{\otimes * r}
$$

is also an upper bound of $T_{m i n}$.

Remark 2: The above result can be strengthened by proving the monotonicity with respect to $k$ of the sequence of upper bounds indexed by $m=\zeta^{k} r$, for $\zeta \geq 2$.

Remark 3: The sufficient condition of Theorem 3 lends itself to be slightly modified so as to cope with the stability analysis of system (1) under the reset condition (3). As a matter of fact, it is enough to replace condition

$$
e^{\mathcal{A}_{i, \otimes m}^{\prime} T} \Pi_{j} e^{\mathcal{A}_{i, \otimes m} T}<\Pi_{i} \quad \forall i \neq j
$$

with

$$
e^{\mathcal{A}_{i, \otimes m}^{\prime} T}\left(S_{j}^{\otimes m}\right)^{\prime} \Pi_{j} S_{j}^{\otimes m} e^{\mathcal{A}_{i, \otimes m} T}<\Pi_{i} \quad \forall i \neq j .
$$

\section{CONDITIONS VIA THE SMR}

Any polynomial $h(x)$ of degree $2 m$ in $x \in \mathbb{R}^{n}$ can be written in a more compact and complete way by using the SMR which was introduced in [14] to establish whether a polynomial is sum of squares of polynomials (SOS) via LMIs.

Indeed, let $x^{\{m\}} \in \mathbb{R}^{d(n, m)}$ be a vector containing a base for the homogeneous polynomials of degree $m$ in $x \in \mathbb{R}^{n}$, where

$$
d(n, m)=\frac{(n+m-1) !}{(n-1) ! m !}
$$

and let us define the set

$$
\mathcal{L}_{m}=\left\{L=L^{\prime}: x^{\{m\}^{\prime}} L x^{\{m\}}=0 \quad \forall x\right\}
$$

whose dimension is given by

$$
d_{\text {par }}(n, m)=\frac{1}{2} d(n, m)(d(n, m)+1)-d(n, 2 m) .
$$

Let $L_{m}: \mathbb{R}^{d_{\text {par }}(n, m)} \rightarrow \mathbb{R}^{d(n, m) \times d(n, m)}$ be any linear parametrization of the set $\mathcal{L}_{m}$. Then, the SMR of $h(x)$ is given by

$$
h(x)=x^{\{m\}^{\prime}}\left(H+L_{m}(\alpha)\right) x^{\{m\}}, \quad \forall \alpha \in \mathbb{R}^{d_{\text {par }}(n, m)}
$$

where $H \in \mathbb{R}^{d(n, m) \times d(n, m)}$ is a suitable constant matrix. See also [16], [17] for details about the SMR, and see [18] where homogeneous polynomial Lyapunov functions and the SMR are exploited for establishing robust stability of uncertain systems with time-varying uncertainties.

Let $\mathcal{A}_{i,\{m\}} \in \mathbb{R}^{d(n, m) \times d(n, m)}$ be the matrix satisfying

$$
\frac{d x^{\{m\}}}{d x} A_{i} x=\mathcal{A}_{i,\{m\}} x^{\{m\}} \quad \forall x
$$


which can be computed with the formula given in [19], [16]. Then we have the following theorem.

Theorem 5: Assume that, for given $T>0$ and positive integer $m$,

$$
\left\{\begin{array}{l}
\exists \Pi_{i}, \alpha_{i}, \alpha_{i, j}: \\
\left\{\begin{array}{l}
\Pi_{i}>0 \quad \forall i \\
\mathcal{A}_{i,\{m\}}^{\prime} \Pi_{i}+\Pi_{i} \mathcal{A}_{i,\{m\}}+L_{m}\left(\alpha_{i}\right)<0 \quad \forall i \\
e^{\mathcal{A}_{i,\{m\}}^{\prime} T} \Pi_{j} e^{\mathcal{A}_{i,\{m\}} T}<\Pi_{i}+L_{m}\left(\alpha_{i, j}\right) \quad \forall i \neq j .
\end{array}\right.
\end{array}\right.
$$

Then, (1) is exponentially stable for every $\sigma(\cdot) \in D_{T}$. Proof. Suppose that (10) holds, and define

$$
v_{i}(x)=x^{\{m\}^{\prime}} \Pi_{i} x^{\{m\}} .
$$

We have that

$$
x^{\{m\}^{\prime}} L_{m}(\alpha) x^{\{m\}}=0 \quad \forall \alpha .
$$

Moreover, it can be shown that

$$
\left(e^{A_{i} t} x\right)^{\{m\}}=e^{\mathcal{A}_{i,\{m\}} t} x^{\{m\}} \quad \forall t \geq 0 .
$$

Hence, it follows that (5) holds, which implies that the system is exponentially stable for every $\sigma(\cdot) \in D_{T}$.

Remark 4: Observe that, for $m=1$, (10) coincides with (8) and (2).

To see that the condition (10) is not more conservative than the condition (8), the following result is noted.

Theorem 6: Assume that, for given $T>0$ and positive integer $m$, (8) holds. Then, (10) holds for the same $T$ and $m$.

Proof. Let $\Pi_{i}$ be such that (8) holds for $T$ and $m$. We now show that there exist $\tilde{\Pi}_{i}, \alpha_{i}, \alpha_{i, j}$ such that (10) holds for the same $T$ and $m$.

Define the homogeneous polynomial Lyapunov functions of degree $2 m v_{i}(x)=\left(x^{\otimes m}\right)^{\prime} \Pi_{i} x^{\otimes m}$. We have that (5) holds with these Lyapunov functions. Now, let us define

$$
\tilde{\Pi}_{i}=K_{0}^{\prime} \Pi_{i} K_{0}
$$

where $K_{0}$ is the matrix satisfying

$$
x^{\otimes m}=K_{0} x^{\{m\}} \quad \forall x .
$$

We have that

$$
\begin{aligned}
v_{i}(x) & =x^{\{m\}^{\prime}} \tilde{\Pi}_{i} x^{\{m\}} \\
\tilde{\Pi}_{i} & >0 .
\end{aligned}
$$

Then, let us define

$$
\Phi_{i}=K_{0}^{\prime}\left(\mathcal{A}_{i, \otimes m}^{\prime} \Pi_{i}+\Pi_{i} \mathcal{A}_{i, \otimes m}\right) K_{0}
$$

We have that

$$
\begin{aligned}
\left.\frac{d v_{i}(x)}{d t}\right|_{\dot{x}=A_{i} x} & =x^{\{m\}^{\prime}} \Phi_{i} x^{\{m\}} \\
\Phi_{i} & <0
\end{aligned}
$$

and

$$
\exists \alpha_{i}: \mathcal{A}_{i,\{m\}}^{\prime} \tilde{\Pi}_{i}+\tilde{\Pi}_{i} \mathcal{A}_{i,\{m\}}+L_{m}\left(\alpha_{i}\right)=\Phi_{i}
$$

because $\mathcal{A}_{i,\{m\}}^{\prime} \tilde{\Pi}_{i}+\tilde{\Pi}_{i} \mathcal{A}_{i,\{m\}}$ and $\Phi_{i}$ are SMR matrices of the same homogeneous polynomial. Lastly, let us define

$$
\Phi_{i, j}=K_{0}^{\prime}\left(e^{\mathcal{A}_{i, \otimes m}^{\prime} T} \Pi_{j} e^{\mathcal{A}_{i, \otimes m} T}-\Pi_{i}\right) K_{0} .
$$

We have that

$$
\begin{aligned}
v_{j}\left(e^{A_{i} T} x\right)-v_{i}(x) & =x^{\{m\}^{\prime}} \Phi_{i, j} x^{\{m\}} \\
\Phi_{i, j} & <0
\end{aligned}
$$

and

$$
\exists \alpha_{i, j}: e^{\mathcal{A}_{i,\{m\}}^{\prime} T} \tilde{\Pi}_{j} e^{\mathcal{A}_{i,\{m\}} T}-\tilde{\Pi}_{i}-L_{m}\left(\alpha_{i, j}\right)=\Phi_{i, j}
$$

because $e^{\mathcal{A}_{i,\{m\}}^{\prime}{ }^{T}} \tilde{\Pi}_{j} e^{\mathcal{A}_{i,\{m\}} T}-\tilde{\Pi}_{i}$ and $\Phi_{i, j}$ are SMR matrices of the same homogeneous polynomial. Therefore, the theorem holds.

Let us indicate with $T_{\{m\}}$ the smallest upper bound of $T_{\min }$ guaranteed by Theorem 5 , i.e.

$$
T_{\{m\}}=\inf \{T \geq 0: \text { (10) holds }\} .
$$

The following result is analogous to Theorem 4 and allows one to calculate $T_{\{m\}}$ via a bisection search where at each iteration the condition (10) is tested.

Theorem 7: Assume that (10) holds for some $T>0$ and positive integer $m$. Then, (10) holds also for $T+\xi$ and $m$ for all $\xi \geq 0$.

Proof. Suppose that (10) holds, and define $v_{i}(x)=$ $x^{\{m\}^{\prime}} \Pi_{i} x^{\{m\}}$. Consider any $\xi \geq 0$. From the second inequality one has that

$$
v_{i}(x(\xi)) \leq v_{i}(x(0)) \quad \forall x(0)
$$

which implies that

$$
e^{\mathcal{A}_{i,\{m\}}^{\prime} \xi} \Pi_{i} e^{\mathcal{A}_{i,\{m\}} \xi} \leq \Pi_{i} .
$$

Pre- and post-multiplying the third inequality of (10) by $e^{\mathcal{A}_{i,\{m\}} \xi}$ and $e^{\mathcal{A}_{i,\{m\}}^{\prime} \xi}$ respectively, one gets that

$$
\begin{aligned}
& e^{\mathcal{A}_{i,\{m\}}^{\prime}(T+\xi)} \Pi_{j} e^{\mathcal{A}_{i,\{m\}}(T+\xi)} \\
< & \Pi_{i}+e^{\mathcal{A}_{i,\{m\}}^{\prime} \xi} L_{m}\left(\alpha_{i, j}\right) e^{\mathcal{A}_{i,\{m\}} \xi} .
\end{aligned}
$$

Lastly, let us observe that

$$
\begin{aligned}
& x^{\{m\}^{\prime}} e^{\mathcal{A}_{i,\{m\}}^{\prime} \xi} L_{m}\left(\alpha_{i, j}\right) e^{\mathcal{A}_{i,\{m\}} \xi} x^{\{m\}} \\
= & \left(e^{A_{i} \xi} x\right)^{\{m\}^{\prime}} L_{m}\left(\alpha_{i, j}\right)\left(e^{A_{i} \xi} x\right)^{\{m\}} \\
= & 0
\end{aligned}
$$

which implies that

$$
e^{\mathcal{A}_{i,\{m\}}^{\prime} \xi} L_{m}\left(\alpha_{i, j}\right) e^{\mathcal{A}_{i,\{m\}} \xi} \in \mathcal{L}_{m} .
$$

Hence,

$$
\exists \tilde{\alpha}_{i, j}: L_{m}\left(\tilde{\alpha}_{i, j}\right)=e^{\mathcal{A}_{i,\{m\}}^{\prime} \xi} L_{m}\left(\alpha_{i, j}\right) e^{\mathcal{A}_{i,\{m\}} \xi}
$$

and, therefore, the theorem holds. 
Remark 5: For the stability analysis of system (1) under the reset condition (3), it is enough to replace, in the statement of Theorem 5, the condition

$$
e^{\mathcal{A}_{i,\{m\}}^{\prime} T} \Pi_{j} e^{\mathcal{A}_{i,\{m\}} T}<\Pi_{i}+L_{m}\left(\alpha_{i, j}\right) \quad \forall i \neq j
$$

with

$$
e^{\mathcal{A}_{i,\{m\}}^{\prime} T} \tilde{S}_{j}^{\prime} \Pi_{j} \tilde{S}_{j} e^{\mathcal{A}_{i,\{m\}} T}<\Pi_{i}+L_{m}\left(\alpha_{i, j}\right) \quad \forall i \neq j
$$

where $\tilde{S}_{j}$ is the matrix satisfying

$$
\left(S_{j} x\right)^{\{m\}}=\tilde{S}_{j} x^{\{m\}} .
$$

Similarly to $T_{\otimes m}$, the upper bound $T_{\{m\}}$ in (12) is characterized by a monotonicity property. Indeed, it can be shown that

$$
T_{\{\zeta m\}} \leq T_{\{m\}} \quad \forall \zeta \geq 1
$$

Analogously to the limit $T_{\otimes *}$ defined for $T_{\otimes m}$, we define the limit $T_{\{*\}}$ for $T_{\{m\}}$, which is also given by

$$
T_{\{*\}}=\lim _{s \rightarrow \infty} T(s)
$$

where

$$
T(s)=\min \left\{T_{\{1\}}, T_{\{2\}}, \ldots, T_{\{s\}}\right\} .
$$

Lastly, it is worth discussing the conservatism of the proposed approach. Since the upper bound $T_{\{m\}}$ in (12) is obtained by exploiting the SMR for establishing positivity of homogeneous polynomials, the conservatism of $T_{\{m\}}$ is related to the possibility of expressing positive homogeneous polynomials as SOS, see for instance [20]-[22], [16].

More specifically, for a fixed value of $m, T_{\{m\}}$ is the best upper bound obtainable with a homogeneous polynomial Lyapunov function of degree $2 m$ provided that the homogeneous polynomials in (5) are positive if and only if they are SOS.

In particular, it is possible to show that $T_{\{m\}}$ coincides with such a best upper bound in the case of second order systems. Moreover, since any positive homogeneous polynomial can be expressed as ratio of two SOS homogeneous polynomials, it is conjectured that $T_{\{*\}}$ coincides with $T_{\min }$.

\section{EXAMPLES}

In this section, some examples are presented to illustrate the usefulness of our computational method. The obtained bounds are compared with the one provided in the pioneering paper [10], i.e.

$$
T_{H M}=\max _{i} \inf _{\alpha>0, \beta>0}\left\{\frac{\alpha}{\beta}:\left\|e^{A_{i} t}\right\| \leq e^{\alpha-\beta t}, \quad \forall t>0\right\} .
$$

In addition, we consider

$$
\begin{gathered}
T_{L B}=\min \left\{T \geq 0 \text { s.t. } \max _{q}\left|\lambda_{q}\left(\prod_{p=1}^{M} e^{B_{p} \tau}\right)\right|<1,\right. \\
\forall \tau>T\}
\end{gathered}
$$

where $\lambda_{q}$ denotes a generic eigenvalue and $\left\{B_{1}, B_{2}, \cdots, B_{M}\right\}$ are matrices corresponding to any permutation among those of the set $\left\{A_{1}, A_{2}, \cdots, A_{M}\right\}$. Of course

$$
T_{L B} \leq T_{\min },
$$

i.e. $T_{L B}$ is a lower bound of the minimum dwell time. Despite all our attempts, we were not able to work out a third order example with $T_{\{*\}}>T_{\min }$.

\section{A. Example 1}

Consider

$$
A_{1}=\left[\begin{array}{cc}
0 & 1 \\
-2 & -1
\end{array}\right], \quad A_{2}=\left[\begin{array}{cc}
0 & 1 \\
-9 & -1
\end{array}\right] .
$$

We get the following upper bounds:

\begin{tabular}{c|c|c}
$m$ & $T_{\otimes m}$ & $T_{\{m\}}$ \\
\hline 1 & 0.6222 & 0.6222 \\
2 & 0.6216 & 0.6079 \\
3 & 0.6207 & 0.6073 \\
4 & 0.6197 & 0.6073
\end{tabular}

In this example $T_{H M}=2.2321$ and $T_{L B}=0$. It turns out that the true minimum dwell time $T_{\text {min }}$ coincides with the $T_{\{*\}}$. Indeed, this is confirmed by finding a switching sequence with $t_{k+1}-t_{k}=0.6073-\epsilon$ yielding a non asymptotically stable system. For instance, it can be easily verified that taking the periodic signal of period $t_{1}+t_{2}$

$$
\sigma(t)= \begin{cases}1, & t \in\left[0, t_{1}\right) \\ 2, & t \in\left[t_{1}, t_{1}+t_{2}\right)\end{cases}
$$

with $t_{1}=0.8800$ and $t_{2}=0.6073$, the associated periodic system $\dot{x}(t)=A_{\sigma(t)} x(t)$ is not asymptotically stable (the maximum modulus of the characteristic multipliers is equal to one). Therefore, $T_{\min }=T_{\{*\}}=T_{\{4\}}=0.6073$.

\section{B. Example 2}

Consider

$$
\begin{gathered}
A_{1}=\left[\begin{array}{cc}
-1 & -1 \\
1 & -1
\end{array}\right], \quad A_{2}=\left[\begin{array}{cc}
-1 & 2 \\
-3 & -1
\end{array}\right], \\
A_{3}=\left[\begin{array}{cc}
1 & 1 \\
-3 & -2
\end{array}\right] .
\end{gathered}
$$

We get the following upper bounds:

\begin{tabular}{c|c|c}
$m$ & $T_{\otimes m}$ & $T_{\{m\}}$ \\
\hline 1 & 0.6437 & 0.6437 \\
2 & 0.6281 & 0.3629 \\
3 & 0.4607 & 0.3510 \\
4 & 0.3747 & 0.3510
\end{tabular}

In this example $T_{H M}=2.9816$ and $T_{L B}=0$. Analogously to Example 1, it can be easily verified that taking the periodic signal of period $t_{1}+t_{3}$

$$
\sigma(t)= \begin{cases}1, & t \in\left[0, t_{1}\right) \\ 3, & t \in\left[t_{1}, t_{1}+t_{3}\right)\end{cases}
$$

with $t_{1}=0.3510$ and $t_{3}=0.4700$, the associated periodic system $\dot{x}(t)=A_{\sigma(t)} x(t)$ is not asymptotically stable (the maximum modulus of the characteristic multipliers is equal to one). Therefore, $T_{\min }=T_{\{*\}}=T_{\{4\}}=0.3510$. 


\section{Example 3}

Consider

$$
A_{1}=\left[\begin{array}{ccc}
-1 & -1 & 1 \\
-1 & -1 & 0 \\
-2 & 1 & -1
\end{array}\right], \quad A_{2}=\left[\begin{array}{ccc}
-1 & 0 & 6 \\
-2 & -1 & -5 \\
0 & 3 & -1
\end{array}\right]
$$

We get the following upper bounds:

\begin{tabular}{c|c|c}
$m$ & $T_{\otimes m}$ & $T_{\{m\}}$ \\
\hline 1 & 1.9135 & 1.9135 \\
2 & 1.9108 & 1.9065 \\
3 & 1.9087 & 1.9023 \\
4 & 1.9070 & 1.8997
\end{tabular}

In this example $T_{H M}=15.7089$ and $T_{L B}=1.8788$. Probably $T_{\{4\}}$ is not tight this time, nevertheless it is expected that one can reach $T_{\min }$ for values of $m$ larger than 4 . Observe that, clearly, $T_{\min } \in\left[T_{L B}, T_{\{4\}}\right]=[1.8788,1.8997]$.

\section{Example 4}

Consider

$$
\begin{gathered}
A_{1}=\left[\begin{array}{ccc}
-1 & 1 & 0 \\
0 & -2 & -1 \\
-1 & 0 & -2
\end{array}\right], A_{2}=\left[\begin{array}{ccc}
-1 & 0 & 1 \\
-1 & -1 & 0 \\
0 & 1 & -1
\end{array}\right], \\
A_{3}=\left[\begin{array}{ccc}
-1 & 0 & 6 \\
-1 & -1 & -5 \\
0 & 1 & -1
\end{array}\right] .
\end{gathered}
$$

We get the following upper bounds:

\begin{tabular}{c|c|c}
$m$ & $T_{\otimes m}$ & $T_{\{m\}}$ \\
\hline 1 & 0.3930 & 0.3930 \\
2 & 0.2616 & 0.0549 \\
3 & 0.0027 & 0.0000 \\
4 & 0.0000 & 0.0000
\end{tabular}

Hence $T_{\{*\}}=0$. In this example $T_{H M}=2.2395$. Of course $T_{\text {min }}=T_{L B}=0$.

\section{CONCLUSIONS}

This paper has addressed stability of switched linear systems under a dwell time constraint. LMI conditions have been proposed to compute upper bounds of the minimum dwell time, based on the use of Kronecker products and the SMR of homogeneous polynomials. The examples show that the exact minimum dwell time can be arbitrarily approached by increasing the degree of the homogeneous polynomial. This is in accordance with our conjecture that the proposed LMI conditions are not conservative for dwell time investigations of switched linear systems. Further work will be devoted to prove this conjecture and to derive upper bounds of the degree of the homogeneous polynomial Lyapunov function required to achieve non-conservatism.

\section{ACKNOWLEDGEMENT}

The authors would like to thank the Associate Editor and the Reviewers for their valuable comments. This work is supported in part by the Research Grants Council of Hong Kong (Grant HKU711208E), the Science Foundation of Ireland (Research Grants 07/PI/I1828 and 07/IN.1/I901) and the Conselho Nacional de Desenvolvimento Científico e Tecnológico - CNPq, Brazil.

\section{REFERENCES}

[1] M. Wicks and R. A. DeCarlo. Solution of coupled lyapunov equations for the stabilization of multimodal linear systems. In American Control Conf., pages 1709-1713, 1997.

[2] M. S. Branicky. Multiple lyapunov functions and other analysis tools for switched and hybrid systems. IEEE Trans. on Automatic Control, 43:475-482, 1998.

[3] M. Johansson and A. Rantzer. Computation of piecewise quadratic Lyapunov functions for hybrid systems. IEEE Trans. on Automatic Control, 43:555-559, 1998.

[4] D. Liberzon and A. S. Morse. Basic problems in stability and design of switched systems. IEEE Control Systems Magazine, 19(5):59-70, 1999.

[5] D. Liberzon. Switching in Systems and Control. Birkhauser, Boston, 2003

[6] J. P. Hespanha. Uniform stability of switched linear systems: extensions of lasalle's principle. IEEE Trans. on Automatic Control, 49:470-482, 2004.

[7] Z. Sun and S. Ge. Switched Linear Systems Control and Design. Springer-Verlag, 2005.

[8] F. Blanchini and S. Miani. Set-theoretic approach. Birkhauser, 2007.

[9] R. Shorten, F. Wirth, O. Mason, K. Wulff, and C. King. Stability criteria for switched and hybrid systems. SIAM Reviews, 49(4):545592, 2007.

[10] A. S. Morse. Supervisory control of families of linear set-point controllers - part 1 : Exact matching. IEEE Trans. on Automatic Control, 41(10):1413-1431, 1996.

[11] J. P. Hespanha. Logic-Based Switching Algorithms in Control. $\mathrm{PhD}$ thesis, Yale University, 1998.

[12] J. C. Geromel and P. Colaneri. Stability and stabilization of continuous-time switched systems. SIAM Journal of Control and Optimization, 45(5):1915-1930, 2006.

[13] R. W. Brockett. Finite dimensional linear systems. John Wiley \& Sons, New York, 1970.

[14] G. Chesi, A. Tesi, A. Vicino, and R. Genesio. On convexification of some minimum distance problems. In European Control Conf., Karlsruhe, Germany, 1999.

[15] F. Wirth. A converse lyapunov theorem for linear parameter varying and linear switching systems. SIAM Journal on Control and Optimization, 44:210-239, 2005.

[16] G. Chesi, A. Garulli, A. Tesi, and A. Vicino. Homogeneous Polynomia Forms for Robustness Analysis of Uncertain Systems. Springer, 2009.

[17] G. Chesi. LMI techniques for optimization over polynomials in control: a survey. to appear in IEEE Trans. on Automatic Control.

[18] G. Chesi, A. Garulli, A. Tesi, and A. Vicino. Homogeneous Lyapunov functions for systems with structured uncertainties. Automatica, 39(6):1027-1035, 2003.

[19] G. Chesi, A. Garulli, A. Tesi, and A. Vicino. Robust analysis of LFR systems through homogeneous polynomial Lyapunov functions. IEEE Trans. on Automatic Control, 49(7):1211-1216, 2004.

[20] G. Hardy, J. E. Littlewood, and G. Pólya. Inequalities: Second edition. Cambridge University Press, Cambridge, 1988.

[21] B. Reznick. Some concrete aspects of Hilbert's 17th problem Contemporary Mathematics, 253:251-272, 2000.

[22] G. Chesi. On the gap between positive polynomials and SOS of polynomials. IEEE Trans. on Automatic Control, 52(6):1066-1072, 2007. 